Zbl Arbeitsmed 2016 $\cdot 66: 55-56$ DOI 10.1007/s40664-015-0083-3

Online publiziert: 21. Dezember 2015

c) Springer-Verlag Berlin Heidelberg 2015

CrossMark

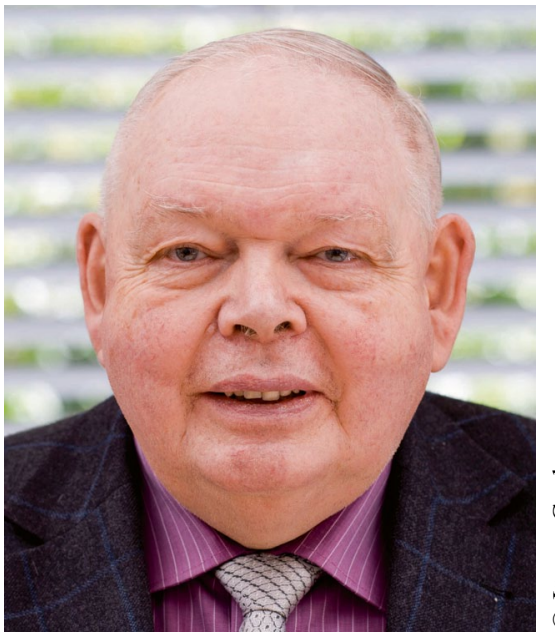

Friedrich Wilhelm Schmahl

Prof. Dr. med. Friedrich Wilhelm Schmahl, bis 2006 Direktor des Instituts für Arbeits- und Sozialmedizin Tübingen, verstarb plötzlich und unerwartet am 20.10.2015 im Alter von 79 Jahren.

Friedrich Wilhelm Schmahl wurde am 28.09.1936 in Velbert geboren. Nach dem Abitur nahm er in Marburg zunächst das Studium der Humanmedizin, der Mathematik und Physik auf, fokussierte sich im Verlauf aber ausschließlich auf Humanmedizin. Er absolvierte das Studium an den medizinischen Fakultäten der Universitäten Erlangen und Heidelberg, wo er im Jahr 1961 bei Professor Hans Schaefer, dem Begründer des Fachs Sozialmedizin in Deutschland, mit der

Dieser Nachruf ist ebenfalls erschienen in: M. Rieger, H. Drexler, S. Hildenbrand (2015) Friedrich Wilhelm Schmahl - Den Menschen im Blick. Arbeitsmed Sozialmed Umweltmed 50:917

\author{
M. Rieger ${ }^{1} \cdot$ H. Drexler ${ }^{2,3} \cdot$ S. Hildenbrand ${ }^{1}$ \\ ${ }^{1}$ Institut für Arbeitsmedizin, Sozialmedizin und Versorgungsforschung, \\ Universitätsklinikum Tübingen, Tübingen, Deutschland \\ 2 Institut und Poliklinik für Arbeits-, Sozial- und Umweltmedizin, \\ Friedrich-Alexander-Universität Erlangen-Nürnberg, Erlangen, Deutschland \\ ${ }^{3}$ Deutsche Gesellschaft für Arbeitsmedizin und Umweltmedizin e. V., München, Deutschland
}

\title{
Friedrich Wilhelm Schmahl
}

\section{Den Menschen im Blick}

Arbeit „Die Wirkung von Adrenalin und anderen gefäßaktiven Substanzen auf isolierte Muskel- und Hautarterien im Vergleich mit den Kreislaufreaktionen im Organismus“ mit der Note „Summa cum laude" promovierte. Nach Abschluss von Studium und Promotion war Friedrich Schmahl ab 1961 zunächst als Medizinalassistent an Kliniken in Heidelberg und Mannheim und ab 1963 bis zum Frühjahr 1966 am physiologischchemischen Institut in Marburg tätig. Als junger Assistenzarzt und Wissenschaftler hat er sich intensiv mit Fragen der Grundlagenforschung auseinander gesetzt und erhielt in dieser Zeit auch ein Ausbildungsstipendium der Deutschen Forschungsgemeinschaft. Nach Jahren der medizinisch-theoretischen Forschung vertiefte Friedrich Schmahl seine klinische Ausbildung als Assistenzarzt an den Medizinischen Universitätskliniken Mainz und Gießen, wo er im Jahr 1972 seine internistische Facharztausbildung und seine Habilitation abschloss und zunächst Universitätsdozent und im Jahr 1975 Professor wurde. Im selben Jahr war er als Gastprofessor an der Harvard University in den USA tätig und brachte von dort wesentliche Impulse für seine spätere wissenschaftliche Tätigkeit im Bereich der Sozialmedizin sowie Arbeits- und Umweltmedizin mit. Diese erweiterten die von ihm in Gießen bearbeiteten, damals wie heute, aktuellen Fragen zur Bedeutung des Fettstoffwechsels für Herzinfarkte um die Betrachtung weiterer Risikofaktoren. Schon damals zeigte sich, dass solche Fragestellungen große
Bedeutung für die Sozial- und Arbeitsmedizin hatten.

1979 berief die Universität Tübingen Professor Schmahl auf den Lehrstuhl für Sozialmedizin des Instituts für Arbeitsund Sozialmedizin, zu dessen Direktor er im Jahr 1981 ernannt wurde. Während seiner über 25 Jahre langen Tätigkeit in Tübingen erweiterte er das Spektrum der arbeitsmedizinischen Forschung am Institut und ergänzte es um vielfältige sozialmedizinische Fragestellungen. Unter anderem lagen ihm die Gesundheit von Menschen mit Migrationshintergrund und die weltweite Gestaltung der medizinischen Versorgung am Herzen. Eine lange Publikationsliste in deutschen und internationalen Fachzeitschriften und unzählige Vorträge bei wissenschaftlichen Tagungen spiegeln seine wissenschaftliche Produktivität und Arbeitskraft wider, aber auch die intensive Förderung seiner Mitarbeiterinnen und Mitarbeiter. Unter seiner Leitung fanden zahlreiche Konferenzen mit Beteiligung von nationalen und internationalen Wissenschaftlern statt, an denen auch viele hochrangige Vertreter von Politik und Wirtschaft teilnahmen.

Sein breites Interesse sowie seine fundierte Wissenschaftlichkeit machten Professor Schmahl zu einem wertvollen Mitglied verschiedener internationaler und nationaler Gesellschaften und Gremien, so z. B. der Deutschen Gesellschaft für Sozialmedizin und Prävention, der Deutschen Gesellschaft für Arbeitsmedizin und Umweltmedizin, der Deutsch-Israelischen Gesellschaft für arbeitsmedizinische und umwelt- 
medizinische Zusammenarbeit, dem Verband Deutscher Betriebs- und Werksärzte, dem Kuratorium der Carl Friedrich von Weizsäcker-Gesellschaft und dem Kuratorium der Theodor-Heuss-Stiftung. Ausgehend von seinem persönlichen und wissenschaftlichen Interesse an "global health“ war Professor Schmahl u. a. dem Deutschen Institut für Ärztliche Mission eng verbunden.

Jahrelang war er Mitglied des inneren Kreises der Pugwash Conferences on Science and World Affairs und hatte somit Teil am Friedensnobelpreis 1995. Auf den Konferenzen thematisierte er die medizinischen Folgen der Verwendung von Atomwaffen und setzte sich aktiv für deren Reduktion ein.

Professor Schmahl war es stets ein Anliegen, dass seine Forschung den Betroffenen selbst zu Gute kam. Seine Kollegen und Mitarbeitenden schätzten insbesondere seine offene, humorvolle und freundliche Art.

Auch nach seiner Emeritierung nahm er mit großem Interesse Anteil an den Geschicken des Tübinger Instituts für Arbeitsmedizin, Sozialmedizin und Versorgungsforschung und an aktuellen Fragen der Arbeits- und Sozialmedizin.

Wir werden ihn stets in ehrender Erinnerung behalten. Unser Mitgefühl gilt seiner Familie.

Für den Vorstand der Deutschen Gesellschaft für Arbeitsmedizin und Umweltmedizin e. V.:

\section{Prof. Dr. med. Hans Drexler}

Erlangen

Präsident der Fachgesellschaft

Im Namen aller Institutsmitarbeitenden:

Prof. Dr. med. Monika A. Rieger

Tübingen

Dr. rer. nat. Sibylle Hildenbrand

Tübingen

\section{Korrespondenzadresse}

\section{Dr. S. Hildenbrand}

Institut für Arbeitsmedizin, Sozialmedizin und Versorgungsforschung

Universitätsklinikum Tübingen, Wilhelmstr. 27, 72074 Tübingen

sibylle.hildenbrand@med.uni-tuebingen.de

\section{Februar 2016}

Berlin 24.-27.02.2016

32. Deutscher Krebskongress

Wiss. Leitung: Prof. Dr. A. Eggert

Auskunft: Frau Renate Babnik, Deutsche Krebsgesellschaft, Kuno-Fischer-Str. 8, 14057 Berlin, Fon: 030/3229329-25, presse@krebsgesellschaft.de, www.dkk2016.de

Wuppertal 27.-28.02.2016

12. Bundesweiter Betriebsärztetag

Wiss. Leitung: Silke Kretzschmar, Dr. rer. medic. Dipl. -Min. Silvester Siegmann

Auskunft: RG Gesellschaft für Information Organisation $\mathrm{mbH}$, Würmstr. 55, 82166 Gräfelfing, Fon: 089 / 8989 948-0,

info@rg-web.de

\section{März 2016}

München 09.-11.03.2016

56. Wissenschaftliche Jahrestagung der Deutschen Gesellschaft für Arbeitsmedizin und Umweltmedizin e.V.

Themen: Ressourcen und Stressoren in der Arbeitswelt, Molekulare Arbeitsmedizin, Betriebliches Gesundheitsmanagement Auskunft: Deutsche Gesellschaft für Arbeitsmedizin und Umweltmedizin e.V., Schwanthaler Straße 73 b, 80336 München,

gsdgaum@dgaum.de,

www.dgaum.de

\section{April 2016}

Mannheim 09.-12.04.2016

122. Kongress der Deutschen Gesellschaft für Innere Medizin

Themen: Minimalinvasive internistische Eingriffe, Von der Differentialdiagnose zur Systemmedizin, Biomarker-stratifizierte Therapie, Der multimorbide Patient, Neurologie trifft Innere Medizin...

Wiss. Leitung: Prof. Dr. Gerd Hasenfuß Auskunft: Frau Silvia Kamangar, Universitätsmedizin Göttingen (UMG), Georg-August-Universität Göttingen, Klinik für Kardiologie und Pneumologie Herzzentrum Göttingen, Robert-Koch-Str. 40, 37075 Göttingen,

Fon: +49551398768,

silvia.kamangar@med.uni-goettingen.de, www.dgim2016.de

\section{Juni 2016}

Deggendorf 29.06.-01.07.2016

17. Forum Arbeitsmedizin in Deggendorf

Wiss. Leitung: Dr. med. Georg Meyer

Auskunft: RG Gesellschaft für Information und Organisation mbH, Würmstr. 55, 82166 Gräfelfing, Fon: 089 / 8989 948-0,

info@rg-web.de

\section{Oktober 2016}

Berlin 25.-28.10.2016

Deutscher Kongress für Orthopädie und Unfallchirurgie - DKOU 2016

Auskunft: Intercongress $\mathrm{GmbH}$, Wilhelmstraße 7, 65185 Wiesbaden,

Fon: 0611/977160

dkou@intercongress.de,

www.dkou.de 